\title{
Cultura de segurança do paciente no Serviço de Atenção Domiciliar
}

\author{
Patient safety culture in the Home Care Service \\ Cultura de seguridad del paciente en el Servicio de Atención Domiciliaria
}

Recebido: 27/12/2021 | Revisado: 02/01/2022 | Aceito: 11/01/2022| Publicado: 13/01/2022

\author{
Mileyse da Silva Acásio \\ ORCID: https://orcid.org/0000-0003-1706-8362 \\ Universidade Federal de Alagoas, Brasil \\ E-mail: miliacacio@gmail.com \\ Isabel Comassetto \\ ORCID: https://orcid.org/0000-0002-2389-9384 \\ Universidade Federal de Alagoas, Brasil \\ E-mail: isabelcomassetto@gmail.com \\ Amauri dos Santos Araujo \\ ORCID: https://orcid.org/0000-0001-7435-5670 \\ Universidade Federal de Alagoas, Brasil \\ E-mail: amauriaraujo.sms@gmail.com \\ Nathalia Comassetto Paes \\ ORCID: https://orcid.org/0000-0003-0746-7810 \\ Centro Universitário Tiradentes, Brasil \\ E-mail: nanacpaes@yahoo.com.br \\ Raíssa Rafaella Santos Moreno da Silva \\ ORCID: https://orcid.org/0000-0002-7265-9352 \\ Universidade Federal de Alagoas, Brasil \\ E-mail: raissarafaella13@gmail.com \\ Maria Elizabete Rodrigues Viana \\ ORCID: https://orcid.org/0000-0003-1290-1431 \\ Universidade Federal de Alagoas, Brasil \\ E-mail: beterviana@gmail.com \\ Amuzza Aylla Pereira dos Santos \\ ORCID: https://orcid.org/0000-0001-6299-7190 \\ Universidade Federal de Alagoas, Brasil \\ E-mail: amuzza.pereira@eenf.ufal.br \\ Diana Hadaça de Lima Araújo Vilela \\ ORCID: https://orcid.org/0000-0002-0042-3535 \\ Universidade Federal de Alagoas, Brasil \\ E-mail: diana.vilela@ifac.edu.br
}

\begin{abstract}
Resumo
Objetivo: Mensurar a percepção da cultura de segurança do paciente no serviço de atenção domiciliar de Maceió. Método: Estudo transversal, desenvolvido com 88 profissionais, que responderam um questionário sociodemográfico e o Safety Attitudes Questionnaire - Short form 2006, validado para o português. A coleta de dados ocorreu entre os meses de novembro de 2018 a janeiro de 2019. Utilizou-se a estatística descritiva e inferencial, considerando significativo pvalor igual ou menor 0,05 . O ponto de corte para avaliação positiva foi $\geq 75$ pontos. Resultados: As médias dos domínios variaram de 69,83 para percepção da Gerência-Secretaria de Saúde a 87,55 para Satisfação no Trabalho. Profissionais com até 10 anos de profissão estão mais satisfeitos com o trabalho. Conclusão: A cultura de segurança do paciente não está fortalecida neste serviço, tendo em vista os baixos escores nos domínios de percepção do estresse, da gerência da secretaria de saúde e de condições de trabalho.

Palavras-chave: Serviços de assistência domiciliar; Segurança do paciente; Cultura; Cultura organizacional; Equipe de assistência ao paciente.

Abstract

Objective: To measure the perception of patient safety culture in the home care service in Maceió. Method: Crosssectional study, developed with 88 professionals, who answered a sociodemographic questionnaire and the Safety Attitudes Questionnaire - Short form 2006, validated for Portuguese. Data collection took place between November 2018 and January 2019. Descriptive and inferential statistics were used, considering a significant $p$-value equal to or less than 0.05 . The cutoff point for a positive assessment was $\geq 75$ points. Results: The means of the domains ranged from 69.83 for the perception of the Management-Secretary of Health to 87.55 for Job Satisfaction. Professionals with up to 10 years of experience are more satisfied with their work. Conclusion: The patient safety culture is not strengthened
\end{abstract}


in this service, given the low scores in the domains of stress perception, health department management and working conditions.

Keywords: Home care services; Patient safety; Culture; Organizational culture; Patient assistance team.

\section{Resumen}

Objetivo: Medir la percepción de la cultura de seguridad del paciente en el servicio de atención domiciliaria en Maceió. Método: Estudio transversal, desarrollado con 88 profesionales, quienes respondieron un cuestionario sociodemográfico y el Safety Attitudes Questionnaire - Short form 2006, validado para portugués. La recolección de datos se realizó entre noviembre de 2018 y enero de 2019. Se utilizó estadística descriptiva e inferencial, considerando un p-valor significativo igual o menor a 0.05 . El punto de corte para una evaluación positiva fue $\geq 75$ puntos. Resultados: Las medias de los dominios variaron de 69,83 para la percepción de la Gerencia-Secretaría de Salud a 87,55 para la Satisfacción Laboral. Los profesionales con hasta 10 años de experiencia están más satisfechos con su trabajo. Conclusión: La cultura de seguridad del paciente no se fortalece en este servicio, dadas las bajas puntuaciones en los dominios de percepción del estrés, gestión del departamento de salud y condiciones laborales.

Palabras clave: Servicios de atención domiciliaria; Seguridad del paciente; Cultura; Cultura de la organización; Equipo de asistencia al paciente.

\section{Introdução}

A preocupação com a Segurança do Paciente (SP) é um tema de âmbito global, ganhando relevância com a publicação do relatório To err is Human: building a Safer Health System, traduzindo "Errar é humano: a construção de um sistema de saúde mais seguro", publicado pelo Institute of Medicine (IOM) no ano de 2000, o qual relatava os efeitos catastróficos referente às vidas humanas, quanto à prejuízos financeiros, resultantes dos erros da assistência de saúde prestada pelos profissionais (Mesquita et al., 2016; Kohn et al., 1999).

Assim, a SP pretende reduzir os danos a um nível aceitável nos serviços de saúde (World Health Organization, 2009), minizar atos inseguros nos processos assistenciais e usar as melhores práticas descritas, para alcançar os melhores resultados para o paciente (Ilha et al., 2016). Tendo a Cultura de Segurança de Segurança do Paciente (CSP) como parte da estruturação geral de uma organização, que se refere à forma como a segurança do paciente é projetada e implementada dentro de uma organização, assim como as estruturas e processos que visam apoiá-los (Pinheiro \& Souza, 2016). A CSP é o produto de valores individuais e de um grupo, atitudes, percepções, competências e padrão de comportamento que determinam o compromisso, o estilo e a proficiência da administração de uma organização saudável e segura (Costa et al., 2018)

Diante deste contexto, incidentes relacionados à SP identificados nos hospitais têm início em outros serviços de assistência de saúde, como a Atenção Primária à Saúde (APS) (Mesquita et al., 2016). Embora o Programa Nacional de Segurança do Paciente (PNSP) exista desde 2013, apenas com a portaria $\mathrm{n}^{\circ} 2.436$ de 21 de setembro de 2017, que a necessidade de implantação de ações para promover a SP no âmbito da APS foi mencionada (Ministério da Saúde, 2017; Raimondi et al., 2019).

Braga et al. (2016) menciona mudanças demográficas e epidemiológicas com o uso racional dos leitos hospitalares, redução dos custos de assistência dos sistemas de saúde e a adoção da lógica no cuidado ancorado na humanização, como tendências em crescimento no cenário global, favorecendo ao desenvolvimento da Atenção Domiciliar (AD). Que insere-se na Rede de Atenção à Saúde (RAS) do Sistema Único de Saúde (SUS) assumindo os princípios e diretrizes deste sistema, que devem ser observados na organização do Serviço de Atenção Domiciliar (SAD) (Braga et al., 2016; Castro et al., 2018).

Em 24 de agosto de 2011, a Portaria GM/MS n 2.029 definiu a AD no SUS e instituiu SAD por meio do Programa Melhor em Casa (PMC), desde então, é considerado um dos programas prioritários da Saúde, dado seu potencial de inovação e resolubilidade no cuidado à saúde. Atualmente, tem como base a Portaria GM/MS no 825, de 25 de abril de 2016, que, além de definir o cuidado no domicílio, regulamenta a constituição e funcionamento do SAD (Ministério da Saúde, 2016).

No processo organizacional de um SAD, inclui-se a identificação do fluxo da oferta dos serviços existentes na RAS, desde a solicitação de inclusão e a admissão do usuário mediante os critérios de elegibilidade, seguida do planejamento assistencial, possibilitado pela avaliação do grau de complexidade, definições sobre a modalidade, recursos e tecnologias de 
cuidado demandadas, incluindo periodicidade dos atendimentos (Castro et al., 2018).

Enfatiza-se que o ambiente domiciliar também é propicio aos riscos para o paciente, como os evidenciados no ambiente hospitalar. Motivado pela discussão mundial sobre a SP, sendo de suma importância conhecer e compreender os fatores que influenciam a cultura organizacional nos diversos serviços de saúde, foi demonstrado que cerca de $13 \%$ dos pacientes que dependiam da AD experimentaram algum tipo de evento adverso (EA) (Blais et al., 2013). A fim de evitar a incidência de casos de negligência, imprudência e imperícia, é de suma importância que os profissionais possuam um conjunto de competências pessoais e profissionais indispensáveis à prestação de cuidados no ambiente domiciliar, visando (Alves \& Aguiar, 2020).

Isto posto, delineou-se como pergunta norteadora para esta pesquisa: Qual é a cultura de segurança do paciente dos profissionais do serviço de atenção domiciliar?

Por conseguinte, tem-se o objetivo mensurar a percepção da cultura de segurança do paciente no serviço de atenção domiciliar de Maceió.

\section{Metodologia}

Pesquisa de abordagem quantitativa, transversal realizada com os profissionais do SAD pertencente à Secretaria Municipal de Saúde do município de Maceió, estado de Alagoas, Brasil. Realizada no período de novembro de 2018 a janeiro de 2019, participaram 90 profissionais da equipe multiprofissional de saúde, que estavam inseridos no serviço do SAD há pelo menos 30 dias e excluídos os que estavam afastados das atividades laborais durante o período de coleta, assim como os que realizaram o preenchimento do instrumento de coleta de dados com taxa de resposta inferior a 65\% (Carvalho \& Cassiani, 2012).

O modelo transversal é utilizado quando a exposição é relativamente constante no tempo e o efeito é crônico. Segundo Bastos e Duquia (2007), a condução de um estudo transversal se dá por 3 etapas: 1- definição de uma população de interesse; 2coleta de dados sobre a população estudada; 3- determinação da presença ou ausência do desfecho e a da exposição para cada um dos indivíduos estudados.

As características do estudo transversal estão em aferir na pesquisa causa e efeito concomitantemente em um espaço de tempo único, sem existir espaço de tempo de acompanhamento dos participantes (Pereira, 2008). A estrutura do estudo transversal é importante fonte de informações para a elaboração do diagnóstico em saúde e posterior análise e intervenção a fim de garantir a melhoria da qualidade de atenção (Paese, 2010).

Aprovada pelo Comitê de Ética em Pesquisa (CEP) da Universidade Federal de Alagoas (UFAL) sob o Parecer Consubstanciado $\mathrm{n}^{\mathrm{o}}$ 3.032.368. Os participantes assinaram o Termo de Consentimento Livre e Esclarecido em duas vias, a pesquisa atendeu às diretrizes das Resoluções n. 466/2012.

A abordagem aos participantes aconteceu no próprio ambiente de trabalho, foi utilizado o Questionário Atitudes de Segurança - Short Form (SAQ), composto por 41 itens, agrupados em seis dimensões: Clima de trabalho em equipe (CTE), Clima de segurança (CS), Satisfação no trabalho (ST), Percepção do estresse (PE), Percepção da gerência (PG) e Condições de trabalho (CT). Os itens sem domínio $(14,33$ a 36) referem-se à percepção do trabalhador quando propõe sugestões relativas à segurança do paciente, à colaboração entre os membros da equipe assistencial e às falhas de comunicação alocados na dimensão comportamentos seguros (Carvalho \& Cassiani, 2012).

Os itens foram respondidos através de escala tipo Likert de cinco pontos, com as seguintes opções: A - Discorda totalmente; B - Discorda parcialmente; C - Neutro; D - Concorda parcialmente; E - Concorda totalmente e X - não se aplica. O escore final compreende valores entre 0 (zero), considerado a pior percepção, e 100, a melhor percepção. Desta forma, os escores passaram a assumir os seguintes valores: discordo fortemente $=0$, discordo levemente $=25$, neutro $=50$, concordo levemente $=75 \mathrm{e}$ concordo fortemente $=100$. Alguns itens apresentam escore reverso, de forma que nestas questões um escore reduzido representa uma atitude mais positiva. Os autores do SAQ consideram uma percepção positiva escores igual ou acima de $75^{(14,15)}$. Após a 
coleta, os dados foram inseridos no software de planilha eletrônica Excel®, para posterior processamento e análise. O processamento de dados foi realizado pelo programa Epi info®, versão 7.2.

Para a análise estatística dos dados foi considerado um nível de significância de 5\% e os valores dos dados foram apresentados com um intervalo de confiança de 95\%. Para descrever o perfil da amostra, utilizou-se a estatística descritiva com cálculos de média e desvio padrão. Para a análise de comparação de grupos com duas categorias utilizou-se o teste não paramétrico Teste U de Mann-Whitney, já para a comparação de grupos com mais de duas categorias foram utilizados o teste não paramétrico Kruskal Wallis e o teste ANOVA.

\section{Resultados}

Dentre os 107 profissionais do SAD pertencente à Secretaria Municipal de Saúde do município de Maceió, 90 (84,11\%) aceitaram participar do estudo. Dos 107, houve perda de seguimento de 10 profissionais que não atenderam aos critérios de inclusão, três recusas e dois questionários não foram devolvidos. Dois participantes apresentaram questionários com taxa de resposta inferior a $65 \%$, os quais precisaram ser descartados dos cálculos das médias.

Desta forma, 88 profissionais responderam corretamente os questionários, sendo que $80(90,9 \%)$ eram do sexo feminino, casadas $40(45,5 \%)$ e $31(35,2 \%)$ tinham até 10 anos de formação. A faixa etária predominante foi de 28 a 37 anos $(46 ; 52,3 \%)$. Dos questionários respondidos, 27 participantes afirmaram que a frequência dos treinamentos foi mensal $(30,7 \%)$. Com relação a outros vínculos empregatícios, $47(53,4 \%)$ participantes relataram não ter outros empregos e $86(97,7 \%)$ afirmaram ter afinidade com a área de trabalho.

Houve predomínio de colaboradores da equipe de enfermagem (51; 57,9\%), seguido de fisioterapeutas (14; 15,9\%), médicos $(06 ; 6,8 \%)$ e outros profissionais $(17 ; 19,3 \%)$, compostos pelas demais categorias profissionais, a saber: administrativo ( $04 ; 4,5 \%)$, assistente social $(02 ; 2,3 \%)$; dentista $(03 ; 3,4 \%)$; farmacêutico $(02 ; 2,3 \%)$; fonoaudiólogo $(01 ; 1,1 \%)$; nutricionista $(03 ; 3,4 \%)$, psicólogo $(01 ; 1,1 \%)$ e terapeuta ocupacional $(01 ; 1,1 \%)$. Os resultados evidenciaram que a maioria dos profissionais tinham pós-graduação concluída e/ou em andamento $(60 ; 68,1 \%)$.

Dentre os seis domínios de segurança avaliados, o domínio Satisfação no Trabalho obteve maior pontuação $(87,55)$, o que indica uma visão positiva do ambiente de trabalho. Contudo, os domínios de percepção da gerência da Secretaria Municipal de Saúde, Percepção do estresse e Condições de Trabalho obtiveram escores menores que 75, que significam percepções negativas do ambiente e da gestão do serviço.

Os resultados da Tabela 1 evidenciam os valores resultantes da análise descritiva do escore geral e por domínios do SAQ:

Tabela 1 - Avaliação dos escores do SAQ, segundo domínios. Maceió, Alagoas. 2019.

\begin{tabular}{lllll}
\hline Domínios & Mín. & Média & Máx. & DP* \\
\hline Clima de Trabalho em Equipe & 16,66 & 82,41 & 100,00 & 14,42 \\
Clima de Segurança & 21,43 & 76,99 & 100,00 & 14,91 \\
Satisfação no Trabalho & 10,00 & 87,55 & 100,00 & 13,95 \\
Percepção do Estresse & 0,00 & 72,72 & 100,00 & 20,54 \\
Percepção da Gerência- Secretaria de & 4,16 & 69,83 & 100,00 & 18,69 \\
Sáude & 37,5 & 75,80 & 100,00 & 15,14 \\
Percepção da Gerência- SAD & 0 & 74,00 & 100,00 & 23,53 \\
Condições de Trabalho & &
\end{tabular}

DP*- Desvio Padrão. Fonte: Banco de dados do pesquisador (2019). 
Em relação ao sexo, o escore médio dos Domínios Clima de Trabalho em Equipe (CTE), Clima de Segurança (CS), Satisfação no Trabalho (ST), Percepção da Gerência (Secretaria de Saúde e SAD) foram maiores para os homens. Referente ao domínio Condição de Trabalho, o escore médio para o sexo masculino foi de 59,37 ( $\mathrm{DP}=21,90$ ), enquanto para o feminino foi de 75,47 ( $\mathrm{DP}=23,31)$, com diferença estatisticamente significativa $(\mathrm{p}=0,0425)$.

Quanto ao tempo de formação, os profissionais que tinham mais de 21 anos de formação e menos de um ano de formação

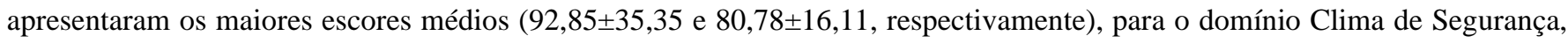

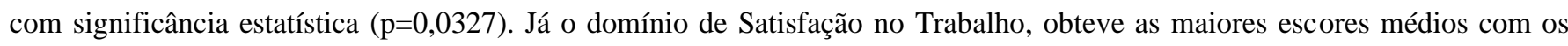

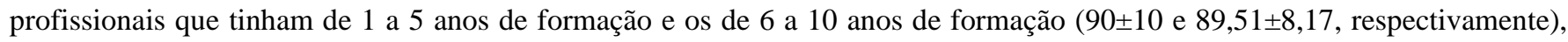
além de significância estatística $(\mathrm{p}=0,0184)$. Esse resultado evidenciou que os profissionais com até 10 anos de tempo de formação apresentaram melhor percepção em relação a visão positiva do local de trabalho. Além disso, os profissionais com menos e mais tempo de formação tiveram os maiores escores para o Clima de Segurança, o que mostra confiança no comprometimento organizacional para a segurança do paciente.

$\mathrm{Na}$ tabela 2, as categorias profissionais foram divididas em 10 grupos, no qual o grupo outros inclui as profissões de fonoaudiólogo, psicólogo e terapeuta ocupacional. Nas comparações das médias pelos domínios do SAQ, mostrou que o pessoal administrativo apresentou o maior escore no domínio Clima de Trabalho em Equipe (91,66), enquanto que os técnicos de enfermagem tiveram maior média nos domínios de Satisfação no Trabalho $(88,92)$ e Percepção da Gerência - SAD $(78,12)$. Para os profissionais de ensino superior, os dentistas tiveram as maiores médias nos domínios de Clima de Segurança $(86,9)$ e Satisfação no Trabalho (95), já os domínios Percepção do Estresse, Percepção da Gerência - Secretaria Municipal de Saúde e Condição de Trabalho tiveram os maiores escores nas categorias farmacêutico $(84,37)$, fisioterapeuta $(77,08)$ e médico $(83,33)$, respectivamente.

Tabela 2 - Escores de percepção da cultura de segurança do paciente por domínios e categoria profissional no SAD-Maceió. Maceió-Al, 2019.

\begin{tabular}{|c|c|c|c|c|c|c|c|c|}
\hline & & $\begin{array}{l}\text { CTE } \\
\text { Média }\end{array}$ & $\begin{array}{l}\text { CS } \\
\text { Média }\end{array}$ & $\begin{array}{l}\text { ST } \\
\text { Média }\end{array}$ & $\begin{array}{l}\text { PE } \\
\text { Média }\end{array}$ & $\begin{array}{l}\text { PG-SMS } \\
\text { Média }\end{array}$ & $\begin{array}{l}\text { PG-SAD } \\
\text { Média }\end{array}$ & $\begin{array}{l}\text { CT } \\
\text { Média }\end{array}$ \\
\hline Categoria Profissional & $\mathrm{N}$ & & & & & & & \\
\hline Administrativo & 4 & 91,66 & 85,71 & 88,75 & 67,19 & 48,95 & 68,75 & 75 \\
\hline Assistente Social & 2 & 45,83 & 48,21 & 40 & 46,87 & 60,41 & 60,41 & 75 \\
\hline Dentista & 3 & 90,27 & 86,90 & 95 & 62,50 & 75,55 & 77,77 & 75 \\
\hline Enfermeiro & 23 & 81,77 & 73,18 & 88,91 & 81,79 & 65,67 & 76,14 & 69,02 \\
\hline Farmacêutico & 2 & 72,91 & 69,64 & 77,50 & 84,37 & 56,25 & 56,25 & 31,25 \\
\hline Fisioterapeuta & 14 & 84,22 & 80,44 & 88,92 & 66,07 & 77,08 & 77,26 & 75 \\
\hline Médico & 6 & 90,27 & 82,34 & 90,83 & 62,50 & 73,61 & 73,61 & 83,33 \\
\hline Nutricionista & 3 & 87,50 & 78,57 & 88,33 & 83,33 & 75 & 77,77 & 75 \\
\hline Técnico de Enfermagem & 28 & 82,32 & 79,06 & 88,92 & 71,42 & 71,42 & 78,12 & 77,67 \\
\hline Outros* & 3 & 69,33 & 60,71 & 80 & 83,33 & 74,99 & 77,77 & 79,16 \\
\hline
\end{tabular}

Clima e Trabalho em Equipe (CTE), Clima de Segurança (CS), Satisfação no Trabalho (ST), Percepção do Estresse (PE), Percepção da Gerência- Secretaria Municipal de Saúde (PG-SMS), Percepção da Gerência- Serviço de Atenção Domiciliar (PG-SAD), Condições de Trabalho (CT).

Outros*: Psicólogo, Fonoaudiólogo, Terapeuta Ocupacional. Fonte: Banco de dados do pesquisador (2019). 
A categoria assistente social apresentou médias negativas em cinco dos seis domínios do SAQ, apenas o domínio de Condição de Trabalho teve escore positivo (75).

Com relação à idade dos profissionais, as faixas etárias com os maiores escores foram acima de 58 anos e de 18 a 27

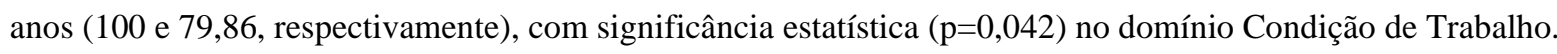

A associação entre os domínios do SAQ e as variáveis categoria profissional, ter outro vínculo empregatício, estado civil, ter especialização, treinamentos e afinidade com a área de trabalho, evidenciou-se que não houve correlação estatisticamente significativa.

\section{Discussão}

No presente estudo, a equipe de enfermagem é em sua maioria do sexo feminino, além disso, constitui a maior parte da força de trabalho nas instituições de saúde, o que justifica ser esta a categoria que mais respondeu ao questionário (Magalhães et al., 2019; Matiello et al., 2016). Dentre os profissionais, 60 (68,1\%) afirmaram possuir pós-graduação em andamento e/ou concluída, o que representa o interesse desses profissionais em sempre realizar capacitação e buscar novos conhecimentos sobre os tratamentos e insumos em sua área de trabalho.

A maioria dos participantes da pesquisa $(47 ; 53,4 \%)$ não possuíam outro vínculo empregatício, o que corrobora com outros estudos realizados no país questionário (Magalhães et al., 2019; Matiello et al., 2016; Luiz et al., 2015; Barbosa et al., 2016). A presença de um único vínculo de trabalho pode ser considerada um fator contribuinte à segurança do paciente, uma vez que, a sobrecarga de trabalho interfere negativamente na assistência prestada, favorecendo a ocorrência de eventos adversos (Magalhães et al., 2019; Rodrigues et al., 2017).

Neste estudo, para $27(30,7)$ profissionais, a frequência de treinamentos foi mensal. Sendo que são consideradas atitudes de segurança que influenciam positivamente a realização de treinamentos em equipe com foco na melhoria da segurança e identificação de perigos e riscos (Rodrigues et al., 2017).

O presente estudo evidenciou que os domínios com escores satisfatórios foram Clima de Trabalho em Equipe, Satisfação no Trabalho, Clima de Segurança e Percepção da Gerência - SAD, com médias de 82,41, 87,55, 76,99 e 75,8, respectivamente.

O Clima de Trabalho em Equipe compreende a qualidade do relacionamento e a colaboração entre os membros de uma equipe que neste estudo se apresentou de forma positiva, com escore de 82,41, tal resultado demonstra que a colaboração e a convivência entre os profissionais é boa neste serviço.

O domínio Satisfação no Trabalho obteve o maior escore dos domínios do SAQ (87,55), o que também foi encontrado em outras pesquisas com valores de 78,7, 81,98, 80,5, 84,60 e 88, respectivamente (Ferno, Radünz, Rosa \& Marinho, 2016; Magalhães et al., 2019; Luiz et al., 2015; Barbosa et al., 2016; Golle et al., 2018). Neste estudo, 86 (97,7\%) participantes declararam que possuem afinidade com o seu trabalho.

A afinidade com o trabalho, ou seja, "gostar do que faz" leva à satisfação laboral, tornando os profissionais satisfeitos profissionalmente com consequente comprometimento com o seu bem-estar e com o do paciente, garantindo-lhes segurança e potencial no desenvolvimento e na produtividade laboral, colaborando com os objetivos e as metas da instituição (Ferno et al., 2016).

O domínio de Clima de Segurança apresentou escore positivo $(76,99)$ e está relacionado ao comprometimento organizacional para segurança do paciente. Outros estudos demonstraram que este domínio obteve escores negativos (Ferno et al., 2016; Luiz et al., 2015; Carvalho et al., 2017). A percepção positiva dos profissionais neste domínio implica que eles não possuem dificuldades de discutir sobre erros com a equipe, se sentem seguros para tratar sobre SP com seus colegas da equipe, além de considerarem que o SAD oferece um cuidado sem riscos aos pacientes.

O domínio de Percepção do Estresse apresentou escore negativo (72,72), o que significa que os profissionais têm 
dificuldade em reconhecer a influência dos fatores estressores na execução do trabalho (Golle et al., 2018). Erros de trabalho, redução da produtividade, sentimentos de desconforto, doença ou mau desempenho da equipe podem ser resultados de falhas em lidar com esses estressores. Portanto, a gestão de estresse é de grande importância, já que ela é um fator limitante para o desenvolvimento das atividades laborais e de grande relevância para a SP, com o objetivo de diminuir os riscos da existência de um cuidado inseguro e de baixa qualidade nas instituições (Ferno et al., 2016; Carvalho et al., 2017).

Embora tenha apresentado um escore positivo sobre a Percepção da Gerência do SAD $(75,80)$, o escore da percepção da gerência da Secretaria de Saúde teve o menor valor em todos os domínios analisados $(69,83)$. Escores positivos para o domínio de percepção da gerência demonstra aceitação e harmonia entre os profissionais, o que favorece o diálogo sobre erros, cultura não punitiva e a realização de treinamentos dos trabalhadores (Golle et al., 2018; Carvalho et al., 2017).

Em contrapartida, escores negativos para este domínio indicam distância entre as equipes assistenciais e a gerência, que pode estar presente devido à cultura punitiva do erro, sem avaliar as circunstâncias em que ocorreu o incidente (Golle et al., 2018). De tal forma, percebe-se a necessidade de avaliação das atitudes apresentadas pelas gerências em relação às questões acerca da segurança do paciente e da relação das mesmas com os profissionais da equipe.

Ressalta-se que no presente estudo, somente dentistas $(75,55)$, fisioterapeutas $(77,08)$ e nutricionistas $(75)$ tiveram percepção positiva para o domínio de Percepção da Gerência da Secretaria de Saúde. Sendo que, a existência de profissionais com melhor percepção de segurança comparados a outros, pode influenciar comportamentos positiva ou negativamente para a prestação de um cuidado seguro, sendo fundamental que para que se promova uma cultura de segurança é necessário que todos os profissionais transmitam a mesma fala e atitudes em prol de uma assistência mais segura (Magalhães et al., 2019). Sendo necessário a obtenção e a consolidação da segurança do paciente, como estratégia para melhoria da qualidade tem como pressuposto o remodelamento do trabalho multiprofissional (Arboit et al., 2020).

O domínio Condições de Trabalho também apresentou escore negativo $(74,00)$, o que demonstra um clima desfavorável a segurança do paciente. Outros estudos nacionais também obtiveram resultados semelhante (Ferno et al., 2016; Magalhães et al., 2019; Luiz et al., 2015; Carvalho et al., 2017). Estes achados podem significar deficiências nos programas de treinamentos, participação não representativa pelos profissionais e na solução de problemas, assim como um fornecimento inadequado de informações para os pacientes, indicando a necessidade de ações de melhoria. Além de que, as políticas relacionadas aos profissionais não são adequadamente priorizadas, gerando consequências para a saúde do trabalhador e pacientes, impactando na segurança do paciente e na qualidade da assistência (Santos et al., 2020; Arboit et al., 2020; Alves \& Aguiar, 2020).

A SP diverge de outro estudo que associou os bons resultados a condições adequadas com ambiente e estrutura de trabalho favorável, incluindo aparatos tecnológicos e materiais (Golle et al., 2018). Pois, quando o profissional não tem acesso à todas informações do paciente, insumos, logística, supervisão adequada, acaba por ter sua pratica assistencial limitada, o que acarreta na perda de sua autonomia e no aumento do risco de incidentes com potencial para causar danos ao paciente (Ferno et al., 2016). No SAD deste estudo não há a categoria de estagiários, o que levou a todos os profissionais a assinalarem o não se aplica a esta questão.

\section{Conclusão}

O uso da SAQ permitiu conhecer a percepção dos profissionais acerca da cultura de segurança do paciente no serviço pesquisada, e revelou que três domínios avaliados tiveram escores negativos, Percepção do Estresse, Percepção da Gerência Secretaria de Saúde e Condição de Trabalho. Logo, para promover um ambiente com cuidado seguro, é necessário identificar os domínios com escores negativos, pois, ao identificar as fragilidades, vislumbra-se possibilidade para maximizar a SP e consequentemente, melhorar a qualidade do serviço prestado.

O estudo mostrou que os profissionais possuem dificuldade em reconhecer os fatores estressores que podem interferir 
no seu trabalho. A distância hierárquica entre a gerência e as equipes de saúde apresenta-se como fator que dificulta o diálogo e a construção de uma cultura não punitiva ao erro. Ao promover condições de trabalho com ambiente agradável, confortável e no qual não falte insumos e tecnologias, e que esteja presente a comunicação entre os profissionais assegura-se a autonomia e a diminuição dos riscos de incidentes no serviço.

Como limitação deste estudo, embora tenha sido utilizada amostragem de conveniência, o corte transversal para obtenção dos dados pode limitar o espectro da análise, contudo, os objetivos propostos foram atingidos. Por fim, recomenda-se a realização de novos estudos, com o principal foco de conhecer e aprofundar-se nas percepções dos profissionais a respeito da cultura de segurança do paciente em outros serviços de saúde, considerando que isto possibilita melhorias na prática e potencialização da segurança aos envolvidos no cuidado.

\section{Referências}

Alves, A. S., \& Aguiar, R. S. (2020). Patient safety at home: an integrative literature review. Research, Society and Development, [S. l.], v. 9, n. 3, p. e181932700, 10.33448/rsd-v9i3.2700. https://rsdjournal.org/index.php/rsd/article/view/2700.

Arboit, Éder L., Bellini, G., Schütz, C. R., Moraes, M. C. S., Kinalski, S. da S., \& Barcellos, R. de A. (2020). Patient safety culture in the multiprofessional perspective. Research, Society and Development, 9(5), e125953088. https://doi.org/10.33448/rsd-v9i5.3088

Barbosa, M. H., Aleixo, T. C. S., Oliveira, K. F. de, Nascimento, K. G. do, Felix, M. M. dos S., \& Barichello, E. (2016). Clima de segurança do paciente em unidades de clínica médica e cirúrgica. Revista Eletrônica De Enfermagem, 18. https://doi.org/10.5216/ree.v18.39763

Bastos, J. L. D., \& Duquia, R. P. (2007). Um dos delineamentos mais empregados em epidemiologia: estudo transversal. Scientia Medica, 17(4), 229-32. http://revistaseletronicas.pucrs.br/fo/ojs/index.php/scientiamedica/article/view/2806/7864.

Blais, R., Sears, N. A., Doran, D., Baker, G. R., Macdonald, M., Mitchell, L., \& Thalès, S. (2013). Assessing adverse events among home care clients in three Canadian provinces using chart review. BMJ Qual Saf. 22:989-997. http://dx.doi.org/10.1136/bmjqs-2013-002039

Braga, P. P., Sena, R. R., Seixas, C. T., Castro, E. A. B., Andrade, A. M., \& Silva, Y. C. (2016). Oferta e demanda na atenção domiciliar em saúde. Ciênc. saúde

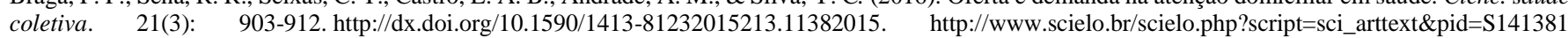
$232016000300903 \& \operatorname{lng}=\mathrm{en}$

Carvalho, R. E. F. L., \& Cassiani, S. H. B. (2012). Questionário Atitudes de Segurança: adaptação transcultural do Safety Attitudes Questionnaire - Short Form 2006 para o Brasil. Rev. Latino-Am. Enfermagem. 20(3). http://dx.doi.org/10.1590/S0104-11692012000300020

Carvalho, R. E. F. L. Arruda, L. P., Nascimento, N. K. P., Sampaio, R. L., Cavalcante, M. L. S. N., \& Costa, A. C. P. (2017). Assessment of the culture of safety in public hospitals in Brazil. Rev. Latino-Am. Enfermagem; 25: e2849. http://www.scielo.br/scielo.php?script=sci_arttext\&pid=S010411692017000100310\&lng=en. http://dx.doi.org/10.1590/1518-8345.1600.2849.

Castro, E. A. B. Leone, D. R. R., Santos, C. M., Gonçalves Neta, F. C. C., Gonçalves, J. R. L., Contim, D. \& Silva, K. L. (2018). Organização da atenção domiciliar com o Programa Melhor em Casa. Rev Gaúcha Enferm. 39:e2016-0002. https://doi.org/10.1590/1983-1447.2018.2016-0002.

Costa D. B., Ramos D., Gabriel C.S., \& Bernardes A. (2018). Cultura De Segurança Do Paciente: Avaliação Pelos Profissionais De Enfermagem. Texto contexto - enferm. 27(3): e2670016. http://www.scielo.br/scielo.php?script=sci_arttext\&pid=S010407072018000300303\&lng=en. http://dx.doi.org/10.1590/0104070720180002670016

Fermo, V. C., Radünz, V., Rosa, L. M., \& Marinho, M. M. (2016). Atitudes profissionais para cultura de segurança do paciente em unidade de transplante de medula óssea. Rev. Gaúcha Enferm. 37(1): e55716. http://dx.doi.org/10.1590/1983-1447.2016.01.55716. http://www.scielo.br/scielo.php?script=sci_arttext\&pid=S1983-14472016000100407\&lng=en.

Golle, L., Ciotti, D., Herr, G. E. G., Aozane, F., Schmidt, C. R., \& Kolankiewicz, A. C. B. (2018). Culture of patient safety in hospital private / Cultura de segurança do paciente em hospital privado. Revista De Pesquisa Cuidado é Fundamental Online, 10(1), 85-89. https://doi.org/10.9789/21755361.2018.v10i1.85-89

Ilha, P., Radünz, V., Tourinho, S. F. V., \& Marinho, M. M. (2016). Segurança do paciente na percepção de acadêmicos de enfermagem. Cogitare Enfermagem. 21(esp):01-10. https://revistas.ufpr.br/cogitare/article/view/43620/pdf

Kohn, L. T., Corrignan, J. M, \& Donaldson, M. S. (1999) To err is human: building a safer health system. $2^{\text {a }}$ ed. Washington: National Academy Press.

Luiz, R. B., Simões, A. L. A., Barichello, E., \& Barbosa, M. H. (2015). Factors associated with the patient safety climate at a teaching hospital. Rev Latino-Am Enfermagem. 23(5):880-7. https://doi.org/10.1590/0104-1169.0059.2627

Magalhães, F. H. L., Pereira, I. C. A., Luiz, R. B., Barbosa, M. H., \& Ferreira, M. B. G. (2019). Clima de segurança do paciente em um hospital de ensino. Rev. Gaúcha Enferm. $\quad$ 40(spe): $\quad$ e20180272. Epub 10-Jan-2019. http://dx.doi.org/10.1590/1983-1447.2019.20180272. http://www.scielo.br/scielo.php?script=sci_arttext\&pid=S1983-14472019000200406\&lng=pt.

Matiello, R. D. C., Lima, E. F. A., Coelho, M. C. R., Oliveira, E. R. A., Leite, F. M. C., \& Primo, C. C. (2016). A cultura de segurança do paciente na perspectiva do enfermeiro. Cogitare enferm. 21(esp):01-09. https://revistas.ufpr.br/cogitare/article/view/45408/pdf 
Research, Society and Development, v. 11, n. 1, e51411125174, 2022

(CC BY 4.0) | ISSN 2525-3409 | DOI: http://dx.doi.org/10.33448/rsd-v11i1.25174

Mesquita, K. O., Silva, L. C. C., Lira, R. C. M., Freitas, C. S. L., \& Lira, G. V. (2016). Segurança do paciente na atenção primária à saúde: revisão integrativa. Cogitare Enfermagem. 21(2):01-08. https://revistas.ufpr.br/cogitare/article/view/45665/28526

Ministério da Saúde (BR). (2016). Secretaria de Atenção à Saúde. Departamento de Atenção Hospitalar e de Urgência. Segurança do paciente no domicílio. Brasília: Ministério da Saúde. http://bvsms.saude.gov.br/bvs/publicacoes/seguranca_paciente_domicilio.pdf.

Ministério da Saúde (BR). (2017). Portaria no 2.436, de 21 de setembro de 2017. Brasilia, DF; 2017. https://bvsms.saude.gov.br/bvs/saudelegis/gm/2017/prt2436_22_09_2017.html

Paese, F. (2010). Cultura de Segurança do Paciente na Atenção Primária de Saúde. Dissertação (Mestrado) - Curso de Pós-graduação em Enfermagem, Universidade Federal de Santa Catarina, Florianópolis, 181 p.

Pereira, M. G. (2008). Epidemiologia: teoria e prática. Guanabara Koogan. 576 p.

Pinheiro, J. P., \& Sousa, U. A. (2016). Safety climate in the operating room: Translation, validation and application of the Safety Attitudes Questionnaire. Revista portuguesa de saúde pública. 2016; 34(2):107-116. https://www.sciencedirect.com/science/article/pii/S0870902515000474. https://doi.org/10.1016/j.rpsp.2015.07.006.

Raimondi, D. C., Bernal, S. C. Z., Oliveira, J. L. C., \& Matsuda, L. M. (2019). Cultura de seguranca do paciente na atencao primaria a saude: analise por categorias profissionais. Rev Gaucha Enferm. 40(esp):e20180133. https://doi.org/10.1590/1983-1447.2019.20180133.

Rodrigues, C. C. F. M., Santos, V. E. P., \& Sousa, P. (2017) Patient safety and nursing: interface with stress and Burnout Syndrome. Rev. Bras. Enferm. 70(5): 1083-1088. http://www.scielo.br/scielo.php?script=sci_arttext\&pid=S0034-71672017000501083\&lng=en. http://dx.doi.org/10.1590/0034-7167-2016-0194

Santos, C. de S. C. S., Abreu, D. P. G., Mello, M. C. V. A. de, Roque, T. da S., \& Perim, L. F. (2020). Evaluation of work overload in the nursing team and the impact on the quality of care. Research, Society and Development, 9(5), e94953201. https://doi.org/10.33448/rsd-v9i5.3201

World Health Organization (WHO) (2009). The conceptual framework for the international classification for patient safety. Technical Report and Technical Annexes. http://www.who.int/patientsafety/taxonomy/icps_full_report.pdf 